\title{
Crystal structure of 4-(1',3'-dioxolane-2'-yl)-3-methyl-1,2,5-oxadiazole-2- oxide, $\mathrm{C}_{6} \mathrm{H}_{8} \mathrm{~N}_{2} \mathrm{O}_{4}$
}

\author{
K. Peters, E.-M. Peters, H. G. von Schnering
}

Max-Planck-Institut für Festkörperforschung. Heisenbergstraße 1. D-70506 Stuttgan. Germany

\section{U. Burkhardt}

Universität Würzburg, Institut für Organische Chemie, Am Hubland, D-97074 Würzburg, Germany and V. Jäger

Universität Stuttgan, Institut für Organische Chemie, Pfaffenwaldring 55, D-70569 Stuttgart, Germany

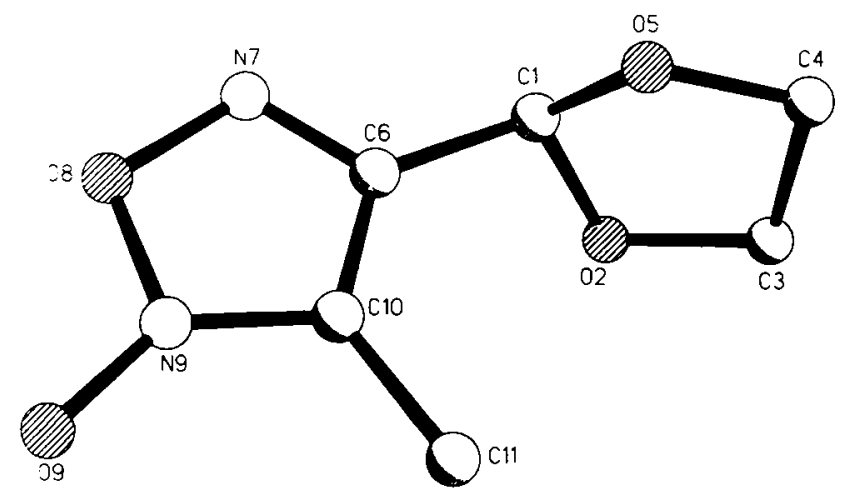

Source of material: The title compound was isolated as a by-product in the Michael addition of nitrous acid to crotonaldehyde, followed by acetalization with ethyleneglycol (see ref. 1). The title compound (mp 371-372 K) was then obtained by recrystallization from methylene chloride (see ref. 2).

$\mathrm{C}_{6} \mathrm{H}_{8} \mathrm{~N}_{2} \mathrm{O}_{4}$, triclinic, $P \overline{1}$ (No. 2), $a=7.210$ (2) $\AA, b=8.296(2) \AA$, $c=7.038(2) \AA, \alpha=104.30(2)^{\circ}, \beta=81.65(2)^{\circ}, \gamma=108.20(2)^{\circ}$, $V=386.5 \AA^{3}, Z=2, R(F)=0.055, R_{w}(F)=0.051$.
Table 1. Parameters used for the X-ray data collection

\begin{tabular}{|c|c|}
\hline $\begin{array}{l}\text { Crystal: } \\
\text { Wavelength: }\end{array}$ & $\begin{array}{l}\text { colorless prism, size } 0.2 \times 0.2 \times 0.6 \mathrm{~mm} \\
\text { Mo } K_{\alpha} \text { radiation }(0.71073 \AA)\end{array}$ \\
\hline$\mu$ : & $1.30 \mathrm{~cm}^{-1}$ \\
\hline Diffractometer: & Siemens P4 \\
\hline Scan mode: & $\omega$ \\
\hline $\mathbf{T}_{\text {measurement: }}$ & $293 \mathrm{~K}$ \\
\hline $2 \boldsymbol{\theta}_{\max }$ : & $55^{\circ}$ \\
\hline $\mathbf{N}(h k l)_{\text {unique: }}$ & 1790 \\
\hline Criterion for $F_{0}$ : & $F_{0}>3 \sigma\left(F_{0}\right)$ \\
\hline $\mathrm{N}(\text { param })_{\text {refined: }}$ & 110 \\
\hline Program: & SHELXTL-plus \\
\hline
\end{tabular}

Table 2. Final atomic coordinates and displacement parameters (in $\AA^{2}$ )

\begin{tabular}{llllll}
\hline Atom & Site & \multicolumn{1}{l}{$x$} & $y$ & $z$ & $U_{\text {iso }}$ \\
\hline H(1A) & $2 i$ & $0.1502(4)$ & $0.6946(4)$ & $-0.0558(5)$ & 0.08 \\
H(3A) & $2 i$ & $0.3036(5)$ & $0.8837(4)$ & $0.4379(5)$ & 0.08 \\
H(3B) & $2 i$ & $0.0986(5)$ & $0.8901(4)$ & $0.3961(5)$ & 0.08 \\
H(4A) & $2 i$ & $-0.0090(5)$ & $0.6054(4)$ & $0.3030(5)$ & 0.08 \\
H(4B) & $2 i$ & $0.1628(5)$ & $0.6035(4)$ & $0.4150(5)$ & 0.08 \\
H(11A) & $2 i$ & $0.6159(5)$ & $0.8099(5)$ & $0.2338(5)$ & 0.08 \\
H(11B) & $2 i$ & $0.8085(5)$ & $0.9262(5)$ & $0.1450(5)$ & 0.08 \\
H(11C) & $2 i$ & $0.7866(5)$ & $0.7305(5)$ & $0.1360(5)$ & 0.08 \\
& & & & & \\
\hline
\end{tabular}

Table 3. Final atomic coordinates and displacement parameters (in $\AA^{2}$ )

\begin{tabular}{|c|c|c|c|c|c|c|c|c|c|c|}
\hline Atom & Site & $x$ & $y$ & $z$ & $U_{11}$ & $U_{22}$ & $U_{33}$ & $U_{12}$ & $U_{13}$ & $U_{23}$ \\
\hline$C(1)$ & $2 i$ & $0.2585(4)$ & $0.7175(4)$ & $0.0216(5)$ & $0.055(2)$ & $0.058(2)$ & $0.051(2)$ & $0.019(1)$ & $-0.004(1)$ & $0.011(2)$ \\
\hline $\mathrm{O}(2)$ & $2 i$ & $0.2811(3)$ & $0.8750(3)$ & $0.1591(3)$ & $0.092(2)$ & $0.047(1)$ & $0.065(2)$ & $0.026(1)$ & $0.022(1)$ & $0.018(1)$ \\
\hline$C(3)$ & $2 i$ & $0.2036(5)$ & $0.8395(4)$ & $0.3465(5)$ & $0.079(2)$ & $0.066(2)$ & $0.053(2)$ & $0.019(2)$ & $0.005(2)$ & $0.012(2)$ \\
\hline$C(4)$ & $2 i$ & $0.1303(5)$ & $0.6448(4)$ & $0.3119(5)$ & $0.069(2)$ & $0.062(2)$ & $0.058(2)$ & $0.014(2)$ & $0.007(2)$ & $0.019(2)$ \\
\hline $\mathrm{O}(5)$ & $2 i$ & $0.2299(3)$ & $0.5878(3)$ & $0.1277(3)$ & $0.074(1)$ & $0.043(1)$ & $0.066(1)$ & $0.016(1)$ & $0.014(1)$ & $0.017(1)$ \\
\hline$C(6)$ & $2 i$ & $0.4403(4)$ & $0.7241(4)$ & $-0.1136(4)$ & $0.058(2)$ & $0.045(2)$ & $0.041(2)$ & $0.016(1)$ & $-0.003(1)$ & $0.009(1)$ \\
\hline$N(7)$ & $2 i$ & $0.4295(4)$ & $0.6936(4)$ & $-0.3024(4)$ & $0.077(2)$ & $0.083(2)$ & $0.050(2)$ & $0.024(2)$ & $-0.005(1)$ & $0.011(2)$ \\
\hline$O(8)$ & $2 i$ & $0.6186(4)$ & $0.7116(3)$ & $-0.3846(3)$ & $0.091(2)$ & $0.089(2)$ & $0.043(1)$ & $0.029(1)$ & $0.008(1)$ & $0.011(1)$ \\
\hline$N(9)$ & $2 i$ & $0.7445(4)$ & $0.7561(3)$ & $-0.2270(4)$ & $0.066(2)$ & $0.046(1)$ & $0.056(2)$ & $0.019(1)$ & $0.007(1)$ & $0.014(1)$ \\
\hline$O(9)$ & $2 i$ & $0.9227(3)$ & $0.7809(3)$ & $-0.2718(4)$ & $0.065(2)$ & $0.075(2)$ & $0.085(2)$ & $0.023(1)$ & $0.026(1)$ & $0.024(1)$ \\
\hline
\end{tabular}


Table 3. (Continued)

\begin{tabular}{lllllllllll}
\hline Atom & Site & $x$ & $y$ & $z$ & $U_{11}$ & $U_{22}$ & $U_{33}$ & $U_{12}$ & $U_{13}$ & $U_{23}$ \\
\hline $\mathrm{C}(10)$ & $2 i$ & $0.6334(4)$ & $0.7633(4)$ & $-0.0620(4)$ & $0.058(2)$ & $0.041(2)$ & $0.045(2)$ & $0.016(1)$ & $0.005(1)$ & $0.014(1)$ \\
$\mathrm{C}(11)$ & $2 i$ & $0.7185(5)$ & $0.8116(5)$ & $0.1300(5)$ & $0.059(2)$ & $0.098(3)$ & $0.059(2)$ & $0.023(2)$ & $-0.008(2)$ & $0.014(2)$ \\
\hline
\end{tabular}

Acknowledgments. We are grateful to the Fonds der Chemischen Industrie and to BMFT/ Bundesgesundheitsamt for financial support, further to $\mathbf{M r}$. M. Frobenius (participant of the undergraduate research programme) for experimental help.

\section{References}

1. Öhrlein, R.; Jäger, V.: 3-Nitrobutanal - Ein neuer Baustein für methylverzweigte Zucker. Tetrahedron Lett. 29 (1988) 6083-6086.

2. Burkhardt, U.; Jäger, V.: Unpublished results.

3. Sheldrick, G. M.: Program Package SHELXTL-plus. Release 4.1. Siemens Analytical X-Ray Instruments Inc., Madison (WI 53719), USA 1990. 